\title{
Differential expression of microRNAs as predictors of glioblastoma phenotypes
}

\author{
Barrie S Bradley ${ }^{1 *}$, Joseph C Loftus ${ }^{2}$, Clinton J Mielke ${ }^{3}$ and Valentin Dinu ${ }^{1}$
}

\begin{abstract}
Background: Glioblastoma is the most aggressive primary central nervous tumor and carries a very poor prognosis. Invasion precludes effective treatment and virtually assures tumor recurrence. In the current study, we applied analytical and bioinformatics approaches to identify a set of microRNAs (miRs) from several different human glioblastoma cell lines that exhibit significant differential expression between migratory (edge) and migrationrestricted (core) cell populations. The hypothesis of the study is that differential expression of miRs provides an epigenetic mechanism to drive cell migration and invasion.

Results: Our research data comprise gene expression values for a set of 805 human miRs collected from matched pairs of migratory and migration-restricted cell populations from seven different glioblastoma cell lines. We identified 62 down-regulated and 2 up-regulated miRs that exhibit significant differential expression in the migratory (edge) cell population compared to matched migration-restricted (core) cells. We then conducted target prediction and pathway enrichment analysis with these miRs to investigate potential associated gene and pathway targets. Several miRs in the list appear to directly target apoptosis related genes. The analysis identifies a set of genes that are predicted by 3 different algorithms, further emphasizing the potential validity of these miRs to promote glioblastoma.

Conclusions: The results of this study identify a set of miRs with potential for decreased expression in invasive glioblastoma cells. The verification of these miRs and their associated targeted proteins provides new insights for further investigation into therapeutic interventions. The methodological approaches employed here could be applied to the study of other diseases to provide biomedical researchers and clinicians with increased opportunities for therapeutic interventions.
\end{abstract}

Keywords: MicroRNA, miR, Glioblastoma, Cell migration, Gene expression, Target prediction, Pathway analysis

\section{Background}

Glioblastoma (GB) is the most common primary central nervous system tumor and accounts for approximately $40 \%$ of all primary malignant brain tumors. GB is a heterogeneous group of tumors associated with a very poor clinical prognosis. The median survival for patients with newly diagnosed glioblastoma is approximately 15 months and declines to approximately 8 months for patients with recurrent glioma $[1,2]$. The 6 -month progression free survival for glioblastoma is less than $20 \%$. The biology of malignant glioma presents significant problems for successful clinical treatment. Chief among these hurdles is the aggressive local invasion of malignant cells

\footnotetext{
* Correspondence: barrie.bradley@asu.edu

${ }^{1}$ Department of Biomedical Informatics, Arizona State University, 13212 East

Shea Boulevard, Scottsdale, AZ 85259, USA

Full list of author information is available at the end of the article
}

from the original tumor. The heightened commitment to migration and reduced proliferation of invasive glioma cells makes complete surgical resection impossible, increases their resistance to chemotherapeutic agents, and reduces the efficacy of radiation treatment, which virtually assures tumor recurrence. Improved clinical treatment will therefore ultimately require a more thorough understanding of the molecular mechanisms that regulate the invasion of glioma cells from primary tumor sites as well as the identification and specific targeting of the critical drivers of glioma invasion.

The pathobiology of GB is characterized by temporal and spatial alterations in gene expression that produce phenotypically distinct cell populations. Necrosis, microvascular proliferation, and increased staining for proliferation markers histopathologically characterize the highly cellular tumor core. In contrast, invasive cells at the 
tumor - normal brain interface exhibit a decrease in the expression of proliferation markers and a relative increase in the expression of pro-apoptotic genes $[3,4]$. Gene expression profiling of laser-captured micro dissected cells from paired GB patient tumor core and invasive edge established an invasion signature of genes differentially expressed in the invasive cell population. This gene set represents potential targets to limit glioma dispersal and decreased therapeutic resistance as the invasion process strongly up-regulates survival signaling pathways [4-6].

The molecular mechanisms regulating the expression of pro-invasive or pro-proliferation signaling proteins are not completely understood. One potential mechanism of post-transcriptional regulation of gene expression is through microRNAs (miRs). miRs are a highly diverse class of small ( 20-22 nucleotides), non-protein coding single stranded RNA molecules that play a central role in a broad range of normal biological processes by dynamically regulating protein expression [7]. miR activity has also been linked to various cancers where miRs can function either as potential oncogenes or as tumor suppressors [8]. A potential role for a number of miRs in GB progression has been reported in recent studies [9-11]. The majority of the studies were performed with cultured glioma cell lines or primary GB patient samples to annotate global changes in miR expression, but did not investigate $\mathrm{miR}$ expression in distinct glioma cell populations. In the current study, we investigated a dataset of miRs collected from a matched pair of migration-restricted glioma cell and migratory glioma cell populations to identify differentially expressed miRs associated with each cell population. We conducted pathway enrichment analysis with these miRs to investigate potential associated gene targets. Signaling effectors regulated by the identified differentially expressed miRs represents a potentially rich set of targets for therapeutic development.

\section{Results}

The differential expression (mean edge cell expression minus mean core cell expression) was down-regulated in 193 (24\%), and up-regulated in $612(76 \%)$ of the 805 miRs in our study. Of the 193 down-regulated miRs, 62 (32\%) exhibited both significant FDR corrected $p$-values and $a \geq 2 x$ fold change, while 131 (68\%) did not. Of the 610 up-regulated miRs, $2(<1 \%)$ exhibited both significant FDR corrected $p$-values and $a \geq 2 \mathrm{x}$ fold change, while $610(>99 \%)$ did not. The results indicate that there is a statistically significant relationship between expression direction (down-regulated vs. up-regulated) and differential expression $p$-value / fold change (Fisher's Exact Test Two-sided $\mathrm{p}<0.001)$.

A plot of the differential expression of these data (volcano scatter plot) illustrates graphically the distribution of miRs that are both significant (two-tailed $t$-test) and meaningful ( $\geq 2 \mathrm{x}$ fold change) (Figure 1 ). Those miRs located both above the FDR corrected $-\log _{10} p$-value (1.69, horizontal dashed line), and a greater than $2 x \log _{2}$ fold change ( $\leq-1.0$ or $\geq 1.0$, vertical dashed lines), are considered both significant and meaningful. Grey colored dots in the volcano plot represent those miRs that are either not significant or do not have a $\geq$ twofold differential expression. Black dots represent those miRs that exhibit both a significant differential expression and $\mathrm{a} \geq 2 \mathrm{x}$ fold change, and thus represent those $64 \mathrm{miRs}$ considered for further study (Table 1).

A total of 64 miRs were identified as exhibiting both significant FDR corrected $p$-values and $a \geq 2 \mathrm{x}$ fold change. Of these $64 \mathrm{miRs}$, migratory edge cell expressions were down-regulated $2 \%$ of the time (mean -0.03 , SD not relevant as only one such data point) and upregulated $98 \%$ of the time (mean 1.85, SD 1.61). Migration-restricted core cell expressions were downregulated $3 \%$ of the time (mean -0.25 , SD 0.04 ) and upregulated $97 \%$ of the time (mean 4.00, SD 1.91). Conversely, for the differential cell population expression, mean edge cell expression minus mean core cell expression, the differential expression was down-regulated $97 \%$ of the time (mean -2.14, SD 0.54) and up-regulated 3\% of the time (mean 1.02, SD 0.18) (Table 2).

Recent studies have demonstrated that many of the significant miRs elucidated in our study have been previously implicated in tumor migration/invasion in other cancers, including malignancies in the brain. For some of our identified miRs however, we were unable to find any prior literature that reported validated gene targets. To address this limitation, we utilized several available algorithms that predict gene targets in silico, such as TargetScan or PicTar. Additionally, resources are available that perform enrichment calculations on representative gene categories or biological pathways. These groups could include such categories as signal transduction, cytoskeletal organization, adhesion, apoptosis, proliferation, or transcription factors [12]. For glioma cell migration, categories such as adhesion and cytoskeletal organization would be important to study further for verification. We turned to these bioinformatics approaches to obtain a wider view of potential genes and pathways that could be targeted by these identified miRs.

We employed the DIANA-mirPath [13] pathway analysis web-server to accomplish both target prediction and enrichment analysis. We used three gene target prediction algorithms in mirPath: TargetScan v5, PicTar 4way, and DIANA MicroT v4 to analyze the datasets as separate jobs (Figure 2). After the gene targets were predicted, mirPath calculated the enrichment of genes in all biological pathways available in the KEGG database. 


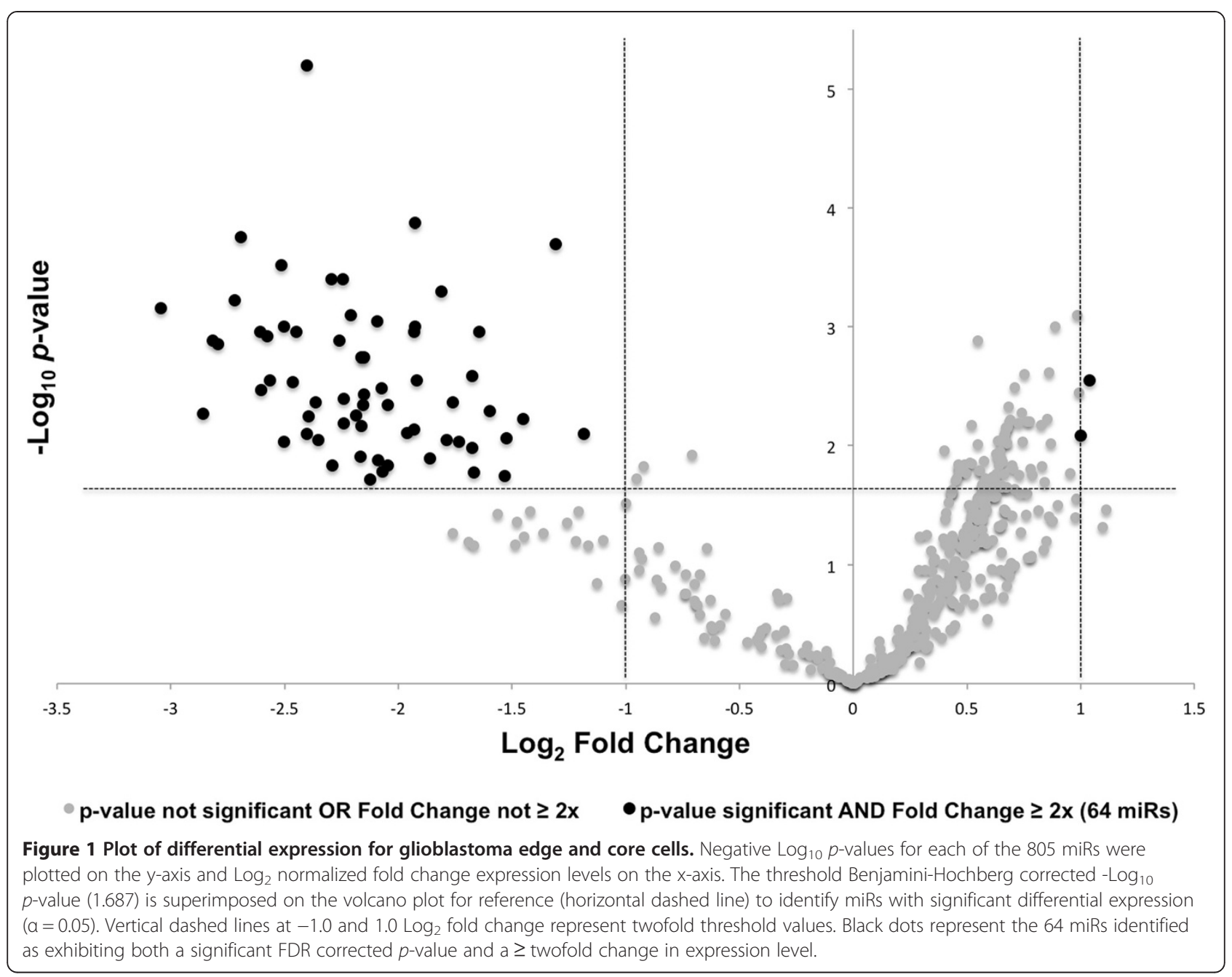

Table 1 miRs identified for study

\begin{tabular}{|c|c|c|c|c|}
\hline hsa-let-7a & hsa-miR-125a-5p & hsa-miR-197 & hsa-miR-26a & hsa-miR-331-3p \\
\hline hsa-let-7b & hsa-miR-125b & hsa-miR-19a & hsa-miR-27a & hsa-miR-365 \\
\hline hsa-let-7c & hsa-miR-130a & hsa-miR-19b & hsa-miR-27b & hsa-miR-424 \\
\hline hsa-let-7d & hsa-miR-140-5p & hsa-miR-20a & hsa-miR-29a & hsa-miR-455-3p \\
\hline hsa-let-7e & hsa-miR-151-3p & hsa-miR-20b & hsa-miR-29b & hsa-miR-574-3p \\
\hline hsa-let-7f & hsa-miR-151-5p & hsa-miR-21 & hsa-miR-29c & hsa-miR-9 \\
\hline hsa-let-7 g & hsa-miR-15a & hsa-miR-22 & hsa-miR-30a & hsa-miR-92a \\
\hline hsa-let-7i & hsa-miR-15b & hsa-miR-221 & hsa-miR-30b & hsa-miR-93 \\
\hline hsa-miR-100 & hsa-miR-16 & hsa-miR-222 & hsa-miR-30c & hsa-miR-99a \\
\hline hsa-miR-103 & hsa-miR-17 & hsa-miR-23a & hsa-miR-30d & hsa-miR-99b \\
\hline hsa-miR-106b & hsa-miR-17* & hsa-miR-23b & hsa-miR-30e & Up-regulated miRs \\
\hline hsa-miR-107 & hsa-miR-181a & hsa-miR-24 & hsa-miR-30e & ebv-miR-BART15 \\
\hline hsa-miR-10b & hsa-miR-181b & hsa-miR-25 & hsa-miR-320 & hsa-miR-548d-5p \\
\hline
\end{tabular}

A total of 62 down-regulated and 2 up-regulated miRs with significant FDR corrected $p$-value and $\geq 2 x$ fold change. 
Table 2 Edge vs. core cell summary statistics for significant miRs

\begin{tabular}{lcrrr}
\hline & \multicolumn{2}{c}{ Frequency } & \multicolumn{2}{c}{ Expression } \\
\hline A. Edge Cells & $\mathbf{n}$ & $\%$ & Mean & SD \\
Down-Regulated & 1 & $2 \%$ & -0.03 & NA \\
Up-Regulated & 63 & $98 \%$ & 1.85 & 1.61 \\
Total miRs & 64 & $100 \%$ & 1.82 & 1.61 \\
B. Core Cells & & & & \\
Down-Regulated & 2 & $3 \%$ & -0.25 & 0.04 \\
Up-Regulated & 62 & $97 \%$ & 4.00 & 1.91 \\
Total miRs & 64 & $100 \%$ & 3.86 & 1.93
\end{tabular}

\section{Differential Expression (Edge - Core)}

$\begin{array}{lrrrl}\text { Down-Regulated } & 62 & 97 \% & -2.14 & 0.54 \\ \text { Up-Regulated } & 2 & 3 \% & 1.02 & 0.18 \\ \text { Total miRs } & 64 & 100 \% & -2.04 & 0.68\end{array}$

Significant miRs identified at $\alpha=0.05$, and utilizing a Benjamini-Hochberg false discovery rate correction.
After analysis, the user is presented with a visualization of any pathways that contained at least one gene. The pathways are ranked according to an enrichment significance score based on a Fisher's combined probability (meta-analysis) test [13]. DIANA-mirPath also provides a union of pathways feature. Using this technique we were able to identify all significantly targeted pathways by the selected miRs. As above, the Fisher's metaanalysis method was used to calculate $p$-values to illustrate the probability that the examined pathway is significantly enriched with gene targets of at least one selected miR [13]. For our list of 64 miRs, 18 enrichment pathways are highly significant $(p<1 \mathrm{E}-16)$. The glioma pathway is ranked as the $7^{\text {th }}$ most significant $(p<1 \mathrm{E}-16)$, and 11 of the 18 highest ranked pathways are cancerrelated, such as endometrial cancer, colorectal cancer, prostate cancer, and bladder cancer. In order to examine the specificity of this approach we conducted the identical union of pathways analysis with a set of 64 randomly selected miRs. For this list of randomly selected miRs, the glioma pathway is not significant $(p=0.08)$. A total

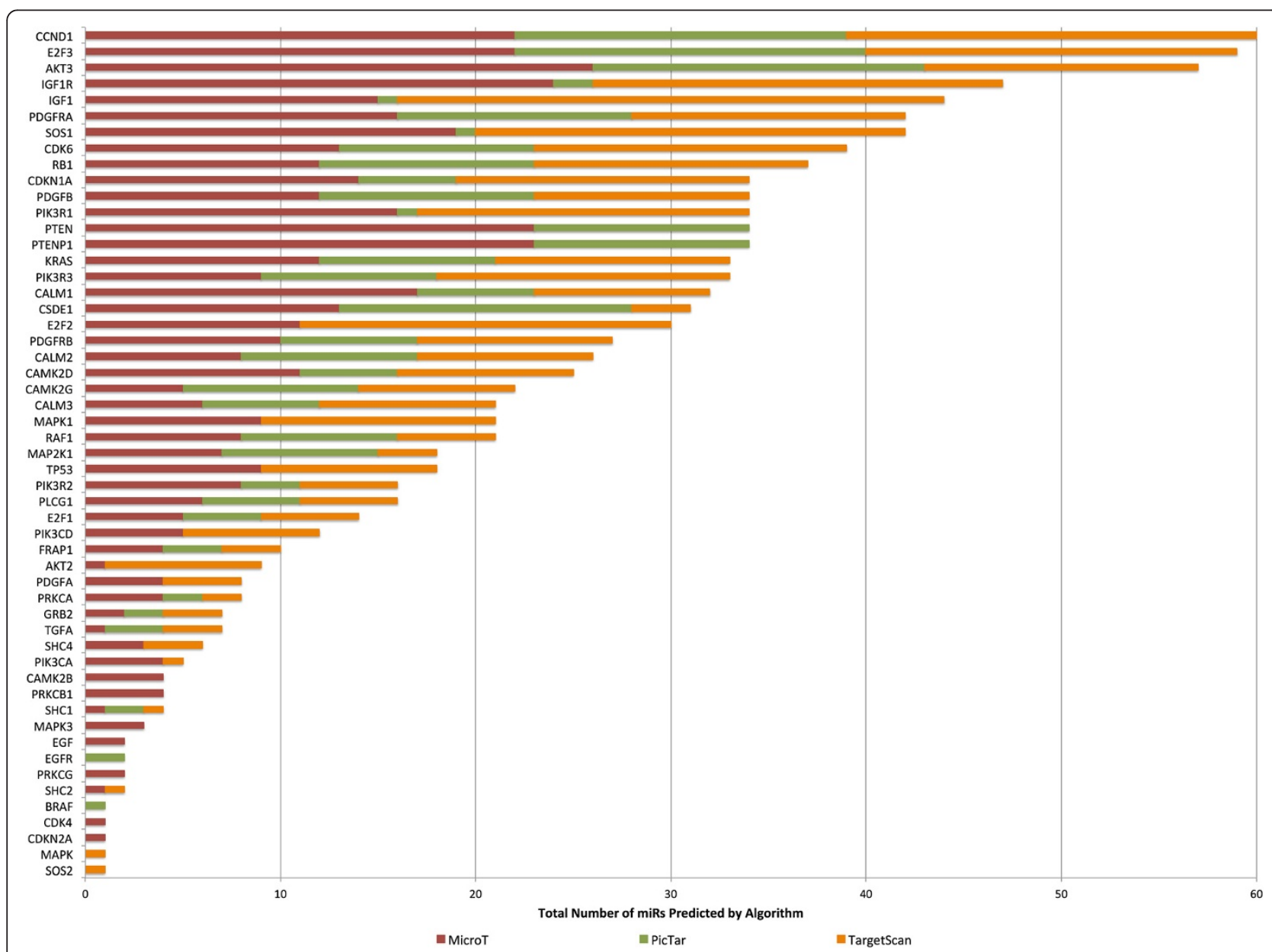

Figure 2 Total number of miRs predicted by algorithm. Illustration of which algorithms predicted the identified gene target by any of the 62 down-regulated miRs. 
of 53 unique genes were identified as potential targets by the three prediction algorithms for glioma pathways (Table 3).

Despite the high rank of the glioma pathway reported by mirPath, we sought a more detailed view of the miRgene interactions. We postulated that some genes might be preferentially targeted by multiple miRs in our dataset. Other studies employing miR pathway analysis favor comparing the results of multiple prediction algorithms to find consensus interactions [12]. Taking a similar approach, we recorded every potential miR-gene interaction among the glioma pathway for all three of the prediction algorithms (Figure 3). We summarized the findings with prediction consensus counts (from consensus of 0 algorithms to consensus of 3 algorithms) to identify the number of algorithms that predicted each miR-gene interaction. We

Table 3 Frequency that a gene is predicted by a miR by number of algorithms

\begin{tabular}{|c|c|c|c|c|c|c|c|}
\hline & \multicolumn{3}{|c|}{ Number of } & \multirow[b]{3}{*}{ Gene } & \multicolumn{3}{|c|}{ Number of } \\
\hline & \multicolumn{3}{|c|}{ Algorithms } & & \multicolumn{3}{|c|}{ Algorithms } \\
\hline Gene & 3 & 2 & 1 & & 3 & 2 & 1 \\
\hline CCND1 & 16 & 4 & 4 & IGF1R & 0 & 22 & 3 \\
\hline E2F3 & 12 & 11 & 1 & SOS1 & 0 & 16 & 10 \\
\hline AKT3 & 11 & 9 & 6 & PIK3R1 & 0 & 16 & 2 \\
\hline PDGFRA & 11 & 4 & 1 & IGF1 & 0 & 15 & 14 \\
\hline PDGFB & 11 & 0 & 1 & E2F2 & 0 & 11 & 8 \\
\hline RB1 & 10 & 2 & 3 & PTEN & 0 & 10 & 14 \\
\hline KRAS & 9 & 3 & 0 & PTENP1 & 0 & 10 & 14 \\
\hline PDGFRB & 7 & 3 & 0 & MAPK1 & 0 & 8 & 5 \\
\hline CDK6 & 6 & 8 & 5 & TP53 & 0 & 8 & 2 \\
\hline CALM1 & 6 & 1 & 12 & PIK3CD & 0 & 5 & 2 \\
\hline CDKN1A & 5 & 8 & 3 & TGFA & 0 & 3 & 1 \\
\hline PIK3R3 & 5 & 6 & 6 & SHC4 & 0 & 3 & 0 \\
\hline CALM2 & 5 & 4 & 3 & PDGFA & 0 & 2 & 4 \\
\hline CAMK2D & 5 & 4 & 2 & PIK3CA & 0 & 1 & 3 \\
\hline RAF1 & 5 & 3 & 0 & $\mathrm{SHC2}$ & 0 & 1 & 0 \\
\hline CALM3 & 5 & 2 & 2 & AKT2 & 0 & 0 & 9 \\
\hline CAMK2G & 5 & 1 & 5 & CAMK2B & 0 & 0 & 4 \\
\hline E2F1 & 4 & 1 & 0 & PRKCB1 & 0 & 0 & 4 \\
\hline CSDE1 & 3 & 4 & 14 & MAPK3 & 0 & 0 & 3 \\
\hline MAP2K1 & 3 & 4 & 1 & EGF & 0 & 0 & 2 \\
\hline PLCG1 & 3 & 3 & 1 & EGFR & 0 & 0 & 2 \\
\hline PIK3R2 & 3 & 2 & 3 & PRKCG & 0 & 0 & 2 \\
\hline FRAP1 & 3 & 0 & 1 & BRAF & 0 & 0 & 1 \\
\hline GRB2 & 2 & 0 & 1 & CDK4 & 0 & 0 & 1 \\
\hline PRKCA & 1 & 1 & 3 & CDKN2A & 0 & 0 & 1 \\
\hline \multirow[t]{2}{*}{ SHC1 } & 1 & 0 & 1 & MAPK & 0 & 0 & 1 \\
\hline & & & & sos2 & 0 & 0 & 1 \\
\hline
\end{tabular}

preferentially focused our attention on interactions unanimously predicted by all three algorithms (score $=3$ ). We then summed the number of unanimous interactions for each gene to assess the enrichment of single genes (Figure 4). This count provided an empirical indication that some genes are potentially targeted by many of the top miRs identified in our analysis. All 3 algorithms predict a glioma pathway gene target by 41 of the 62 down-regulated miRs in our study (Table 4).

\section{Discussion}

Increased miR expression results in decreased messenger RNA (mRNA) expression, which in turn leads to decreased protein expression. Conversely, decreased miR expression could result in increased target mRNA expression, which in turn could lead to increased target protein expression. In the current study, we report the identification of a set of $62 \mathrm{miRs}$ that exhibit statistically significant negative (down-regulated) differential expression in the migratory (edge) cell population relative to the corresponding expression in the matched migration-restricted (core) cell population. Bioinformatics analysis of potential targets of these down-regulated miRs produced a set of genes linked to regulation of apoptosis. Genes targeted by the downregulated miR set have potential for increased expression in the invasive cell population and therefore represent potential therapeutic targets to limit glioma progression.

To begin our investigation of our miRs, we conducted an extensive literature review of verified gene targets relevant to cancer. The let-7 family of miRs is well represented in our results. Let-7 was one of the first two miRs identified and was shown to be a critical regulator of developmental timing [14]. The sequence of let-7 was later discovered in the fruit fly and human genomes via BLAST search and became the first recognized miR in humans [15]. Interestingly, humans have 10 mature let-7 isoforms that are produced from 13 distinct precursor sequences located at different locations in the genome [16]. Eight let-7 family members were present in the set of 62 significant down-regulated miRs in the migratory cell invasive population.

Let-7 members are widely considered critical tumor suppressors that, when lost, can alter cell growth and cancer progression [17]. In glioblastoma, transfection of let-7 $g$ into U251 and U87 glioblastoma cells reduced the expression of Ras family proteins modulating proliferation and invasiveness [18]. Notably, increased expression of let-7 inhibited in vitro proliferation and reduced tumor size in murine xenografts. Other studies have validated Ras as a target for let-7 family members [19]. In addition to let-7, our analysis also indicated that Ras proteins are potential targets for other miRs in our study: $m i R-16$, mir-27b, miR-30c and miR-15b (Figure 3). It is well appreciated that Ras is an integral signaling constituent of many 


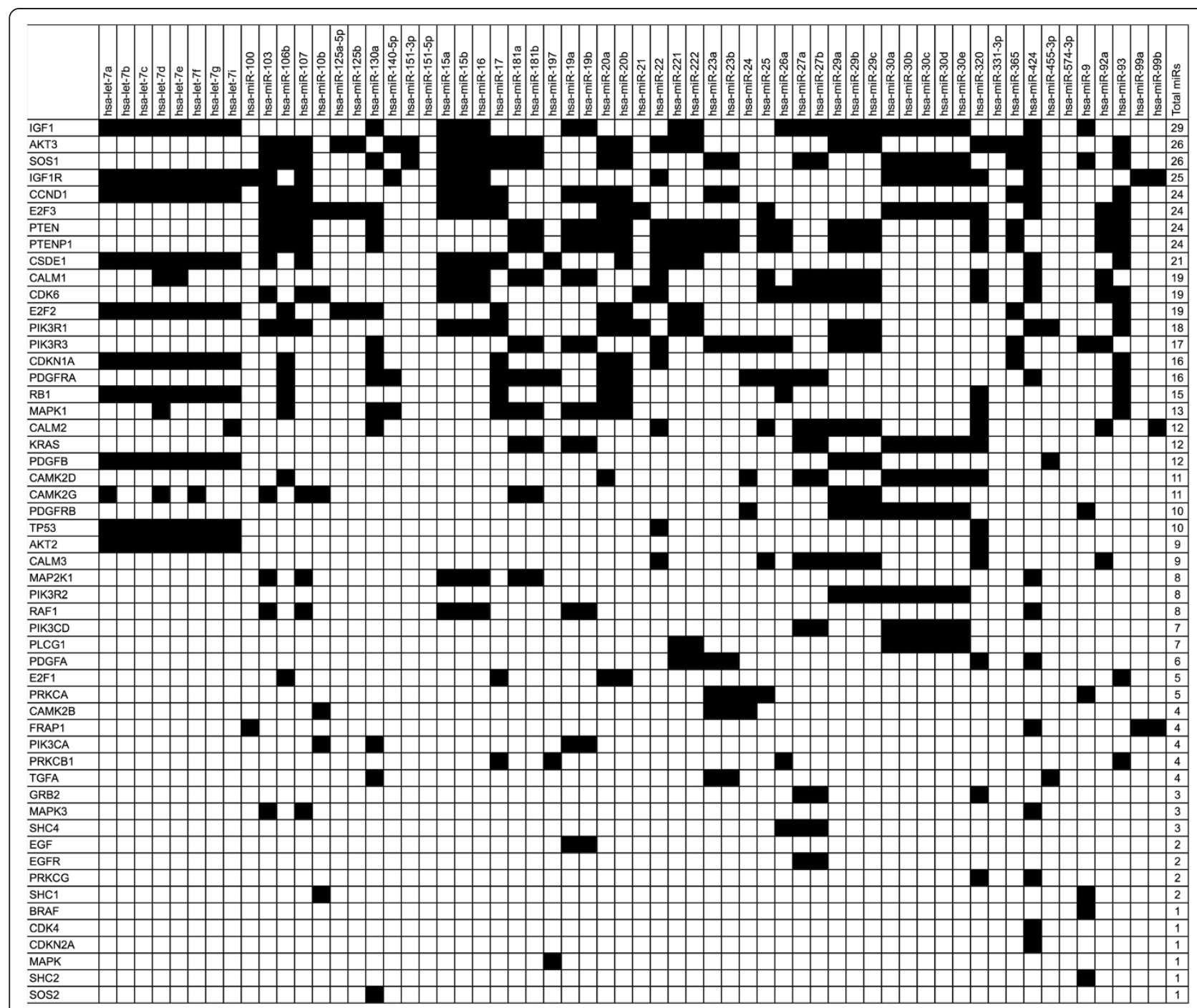

Figure 3 Genes predicted by miR. Illustration of the occurrences that a miR predicts one of the glioma pathway genes by any of the 3 target prediction algorithms.

growth factor receptor pathways and that alterations in several growth factor receptor pathways, including EGFR and PDGFR, are a dominant characteristic of glioblastoma. As Ras signaling has been demonstrated to modulate glioblastoma cell proliferation [20], the loss of these four miRs, along with let-7, are likely to lead to altered Ras expression and activity. Target analysis also revealed that PDGFB is potentially targeted by many miRs in the study that together with PDGFR amplification provides a mechanism to potentiate tumor growth [21].

Two of the miRs in our study have been directly tied to neurological malignancies. Let-7e has been shown to inhibit neuroblastoma proliferation by targeting the MYC oncogene [22]. Meanwhile, miR-181b has been strongly implicated as a tumor suppressor in glioblastoma. Substantial down-regulation of $m i R-181 a$ and $m i R-181 b$ has been observed in both human glioma samples and in established glioma cell lines [23]. Expression of miR-181 was abundant in normal brain tissue, but dropped substantially with increasing WHO grades [23]. Notably, transfection of miR-181b into glioblastoma cells significantly inhibited cell invasion in an in vitro matrigel invasion assay and increased apoptosis in the transfected cells [23].

Several miRs in our list appear to directly target apoptosis related genes. The anti-apoptotic protein BCL2 is a validated target of miR-181b [24] and miR-16 [25]. Furthermore, BCL-XL is degraded by let-7c [20]. Elevated levels of these proteins desensitize cells to apoptosis, and thus it is likely that loss of these relevant miRs in migratory edge cells may cause increased expression of genes and lead to inhibition of apoptosis.

From the pathway enrichment analysis, we find that cyclin D1 (CCND1) stands out as a prominent target of a number of the miRs identified in this study (Figures 2, 


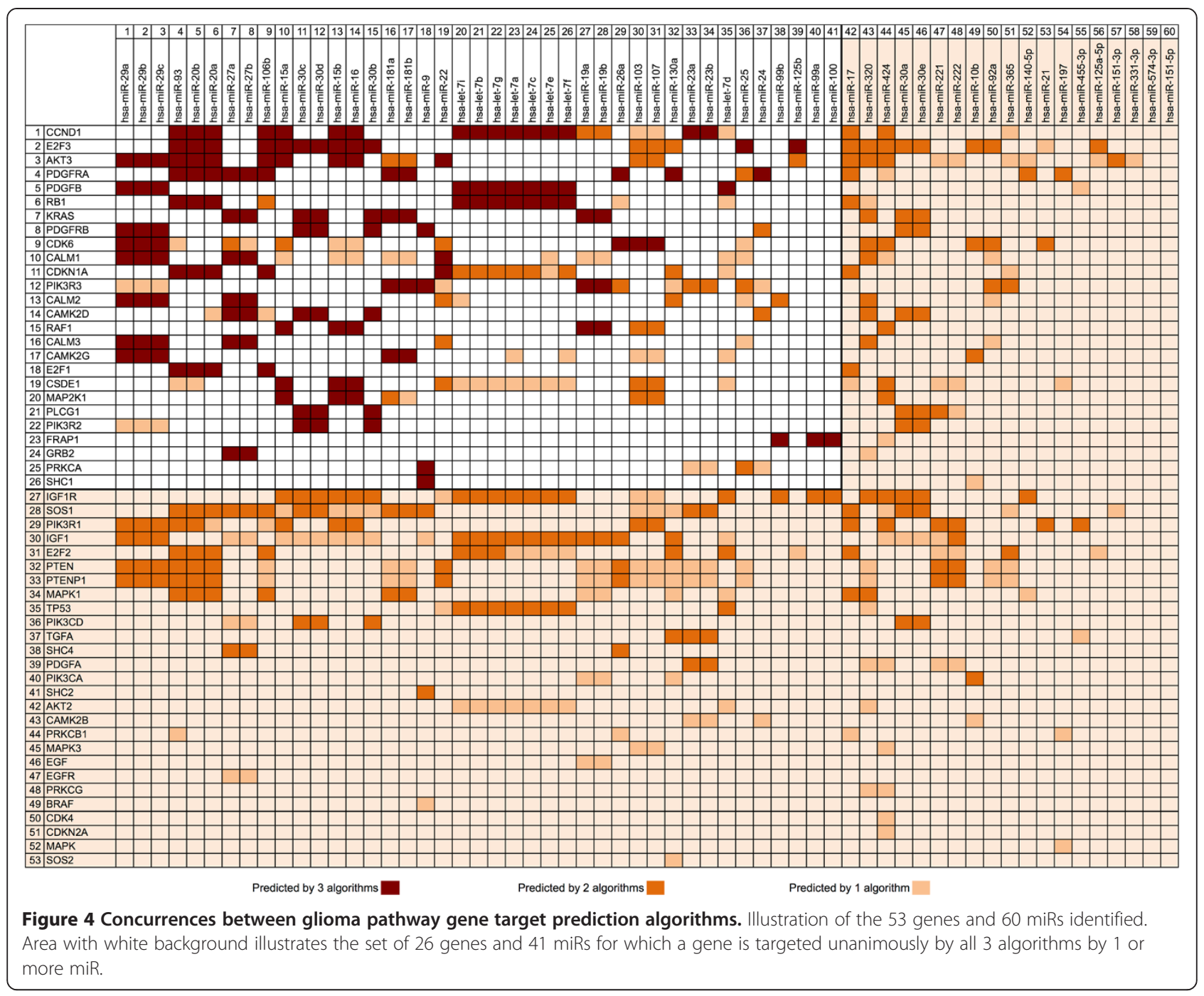

3 and 4). Overexpression of the cyclin D1 protein is associated with tumorigenesis and is associated with poor outcome in a variety of cancers [26]. Cyclin D1 is a critical gene involved in the cell cycle control pathway. It is a regulatory subunit for the CDK4 and CDK6 proteins, and these kinases form active complexes that are required for a cell to progress from $\mathrm{G} 1$ to $\mathrm{S}$ phase. Cyclin D1 also binds to the retinoblastoma protein (RB1). RB1 is itself highly targeted by many of the miRs in our analysis (Figures 2, 3 and 4). The RB1 protein, on the other hand, opposes G1/S checkpoint transitions. It is thus interesting that both of these proteins are predicted targets by the same miRs in the identified set. This suggests a tightly controlled feedback loop that precisely regulates the balance between either cell cycle progression, or arrest, at G1. Perturbations of this balance lead to alterations in cell proliferation.

Mutations or aberrant expression of proteins in the cell cycle pathway have been associated with many cancers. In glioblastoma cell lines, it has been reported that cyclin D1 overexpression promotes invasiveness in vitro [27]. Furthermore, silencing cyclin D1 expression with siRNA inhibited invasion and apoptosis. In vivo, abnormalities in the cell cycle pathway are well recognized. In primary GB, the function of the tumor suppressor proteins p16-INK4A and p14-ARF is affected, whereas in secondary GB it is observed that CDK4 is amplified or RB1 is lost [27]. We speculate that loss of miRs targeting these proteins may lead to an overexpression of cyclin D1, which can deregulate the cell cycle in concordance with these other pathway abnormalities observed in vivo. Notably, our analysis identified a number of miRs that have been previously validated as targeting cyclin D1 including let7a-7f [28] as well as mir15 and miR16 [29]. If many of the other target predictions are valid, then we postulate these miRs may form an integral network involved in regulation of the cell cycle. Because cyclin D1 and RB1 are co-predicted by many of the same set of miRs, 
Table 4 Frequency that a miR predicts a gene by number of algorithms

\begin{tabular}{llllllll}
\hline & \multicolumn{3}{c}{ Number of } & & & \multicolumn{3}{c}{ Number of } \\
\cline { 2 - 4 } & \multicolumn{3}{c}{ Algorithms } & & Algorithms \\
\hline miR & 3 & 2 & 1 & miR & 3 & 2 & 1 \\
hsa-miR-29a & 8 & 4 & 2 & hsa-miR-107 & 1 & 6 & 7 \\
hsa-miR-29b & 8 & 4 & 2 & hsa-miR-130a & 1 & 6 & 7 \\
hsa-miR-29c & 8 & 4 & 2 & hsa-miR-23a & 1 & 4 & 4 \\
hsa-miR-93 & 7 & 6 & 3 & hsa-miR-23b & 1 & 4 & 4 \\
hsa-miR-20b & 7 & 6 & 1 & hsa-let-7d & 1 & 3 & 9 \\
hsa-miR-20a & 7 & 5 & 2 & hsa-miR-25 & 1 & 3 & 6 \\
hsa-miR-27a & 7 & 3 & 3 & hsa-miR-24 & 1 & 2 & 3 \\
hsa-miR-27b & 7 & 2 & 4 & hsa-miR-99b & 1 & 2 & 0 \\
hsa-miR-106b & 6 & 4 & 4 & hsa-miR-125b & 1 & 1 & 1 \\
hsa-miR-15a & 6 & 3 & 3 & hsa-miR-99a & 1 & 1 & 0 \\
hsa-miR-30c & 6 & 3 & 1 & hsa-miR-100 & 1 & 1 & 0 \\
hsa-miR-30d & 6 & 3 & 1 & hsa-miR-17 & 0 & 11 & 2 \\
hsa-miR-15b & 6 & 2 & 4 & hsa-miR-320 & 0 & 10 & 8 \\
hsa-miR-16 & 6 & 2 & 4 & hsa-miR-424 & 0 & 9 & 10 \\
hsa-miR-30b & 6 & 2 & 2 & hsa-miR-30a & 0 & 9 & 1 \\
hsa-miR-181a & 4 & 4 & 3 & hsa-miR-30e & 0 & 9 & 1 \\
hsa-miR-181b & 4 & 3 & 4 & hsa-miR-221 & 0 & 4 & 5 \\
hsa-miR-9 & 4 & 2 & 2 & hsa-miR-222 & 0 & 4 & 5 \\
hsa-miR-22 & 3 & 6 & 3 & hsa-miR-10b & 0 & 4 & 2 \\
hsa-let-7i & 3 & 5 & 3 & hsa-miR-92a & 0 & 3 & 5 \\
hsa-let-7b & 3 & 5 & 2 & hsa-miR-365 & 0 & 2 & 6 \\
hsa-let-7 g & 3 & 5 & 2 & hsa-miR-140-5p & 0 & 2 & 2 \\
hsa-let-7a & 3 & 4 & 4 & hsa-miR-21 & 0 & 2 & 1 \\
hsa-let-7c & 3 & 4 & 3 & hsa-miR-197 & 0 & 1 & 3 \\
hsa-let-7e & 3 & 3 & 5 & hsa-miR-455-3p & 0 & 1 & 2 \\
hsa-let-7f & 3 & 4 & 4 & hsa-miR-125a-5p & 0 & 1 & 2 \\
hsa-miR-19a & 3 & 2 & 6 & hsa-miR-151-3p & 0 & 1 & 1 \\
hsa-miR-19b & 3 & 2 & 6 & hsa-miR-331-3p & 0 & 0 & 1 \\
hsa-miR-26a & 2 & 5 & 2 & hsa-miR-574-3p & 0 & 0 & 0 \\
hsa-miR-103 & 1 & 6 & 7 & hsa-miR-151-5p & 0 & 0 & 0 \\
\hline & & & & & & &
\end{tabular}

they may be appropriate targets for validation (Figures 2, 3 and 4 ).

Also among the miRNAs that was significantly downregulated in the invasive cell population was miR-23b. A previous report demonstrated that miR-23b targets a set of genes associated with tumor invasion and metastasis [30] implicating its loss in facilitating tumor progression. Similarly, a recent report has demonstrated that miR-23b directly targets PIK3R3 [31] substantiating its identification and predicted targeting of PIK3R3 in the current analysis.

\section{Conclusions}

Much progress in the understanding of miRs has been made over the past decade and research is identifying important functions for miRs in cancers such as glioblastoma. It is recognized that alterations in expression of miRNAs can significantly alter the proliferation and invasiveness of cells. Indeed, an increasing number of miRs have been validated as components of cancerdriving pathways. In this study we have applied a set of statistical and informatics tools and approaches, such as $t$-test, FDR, volcano plot, consensus miR target prediction and pathway analysis, to explore the role of miRs in glioblastoma. Approaches similar to the ones described here, that combine bioinformatics analysis of experimentally generated data with in silico miR target prediction and pathway enrichment analysis can be applied to other diseases to provide biomedical researchers and clinicians with increased opportunities for therapeutic interventions.

\section{Methods \\ Data collection}

RNA extraction and miR microarray profiling from matched sets of migratory (edge) and migration-restricted (core) cell populations of seven different glioblastoma cell lines has been described in detail [32]. The data were normalized to eliminate or reduce the potential for fluorescent intensity level bias. Control data was identified and removed before final analysis. The resulting cleaned dataset used in this study comprised 805 human miRs.

\section{Data analysis}

A two-tailed $t$-test statistic $(\alpha=0.05)$ and corresponding $p$-value was calculated for all miRs. SAS 9.2 was used for all statistical analysis. Statistical output for each analyzed miR included tests for normalcy to ensure appropriateness of analytical techniques. False discovery rate (FDR) correction was calculated using a Benjamini-Hochberg correction. A fold change method was used to identify differentially expressed miRs. This method evaluates the $\log$ ratio between two conditions; in this case the $\log _{2}$ normalized expression levels of edge cell populations (migration cells) compared to core cell populations (migration-restricted cells). A twofold difference was used to identify meaningful differentially expressed miRs. In the transformed log scale, a twofold change corresponds to a 1.0 gene signal expression change. As this is not a true statistical test, no confidence levels can be attributed to the differentially expressed or non-differentially expressed miRs.

After calculation of $p$-values, FDR corrections, and log values, the data were further analyzed to identify miRs that exhibited both a significant corrected $p$-value and a greater than twofold change in expression level in the migratory edge cells relative to the migration restricted 
core cells. A total of 64 miRs satisfy the criteria of significant corrected $p$-value and $\geq$ twofold change in expression level. This subset of the data (64 miRs) represents the specific miRs identified for further study. Descriptive statistics were calculated for each of these $64 \mathrm{miR}$ core and edge cell sample expression levels. Each miR was then assessed for dysregulation as either up-regulated (positive expression level) or down-regulated (negative expression level).

A volcano scatter plot method was used to graphically illustrate the relationship between significance levels for differentially expressed miRs. Negative $\log _{10} p$-values for each of the 805 miRs were plotted on the $y$-axis and $\log _{2}$ normalized fold change expression levels on the $\mathrm{x}$ axis. The threshold Benjamini-Hochberg corrected - $\log _{10}$ $p$-value (1.687) was superimposed on the volcano plot for reference (horizontal dashed line) to identify miRs with significant differential expression $(\alpha=0.05)$. In the resulting plot, miRs with a $-\log _{10} p$-value of greater than the threshold corrected - $\log _{10} p$-value (1.687), and a fold change of greater than twofold, comprise the $64 \mathrm{miRs}$ identified for further study.

\section{Target prediction and pathway enrichment analysis}

We employed the DIANA-mirPath website [13] for target prediction and pathway enrichment analysis for the 62 down-regulated miRs identified in the study. As the focus of the study is in migratory glioblastoma cells, we concentrated the majority of our effort on the down-regulated miRs (62) and included the up-regulated miRs (2) only as overall statistical measures in the analysis. We used three gene target prediction algorithms in mirPath: TargetScan v5, PicTar 4-way, and DIANA MicroT v4 to analyze the datasets as separate jobs. The mirPath tool includes a pathways union feature (A Posteriori), which we used to identify all significantly targeted pathways by the selected miR. The mirPath server performs the enrichment analysis and calculates the significance levels ( $p$-values) for each selected miR. The tool then calculates a merged pvalue for each pathway based on a Fisher's combined probability test statistic $\left(X^{2}\right)$. The results from this metaanalysis method depict the probability that the examined pathway is significantly enriched with gene targets of at least one selected miR. We conducted this analysis for both our identified list of $64 \mathrm{miRs}$, as well as for a list of 64 randomly selected miRs in order to assess the specificity of our results.

\section{Availability of supporting data}

Supporting data will be made available upon request.

\footnotetext{
Abbreviations

DIANA: DNA intelligent analysis; FDR: False discovery rate; GB: Glioblastoma; Hsa: Homo sapiens; KEGG: Kyoto encyclopedia of genes and genomes; miR: microRNA; SD: Standard deviation.
}

\section{Competing interests}

The authors declare that they have no competing interests.

\section{Authors' contributions}

BSB is the lead writer and conducted all data processing and analysis, JCL provided the experimental biological data and helped write the manuscript, CJM conducted pathway enrichment analysis, VD oversaw the study and provided overall guidance. All authors read and approved the final version of the manuscript.

\section{Acknowledgements}

This research was supported in part by grants from the Virginia G. Piper Foundation, ASU and Mayo Clinic (VD). This work was supported in part by $\mathrm{NIH}$ grants R01 CA103956 (JCL) and P50 CA108961 (JCL).

\section{Author details}

${ }^{1}$ Department of Biomedical Informatics, Arizona State University, 13212 East Shea Boulevard, Scottsdale, AZ 85259, USA. ${ }^{2}$ Biochemistry and Molecular Biology, Mayo Clinic Arizona, 13400 E. Shea Boulevard, Scottsdale, AZ 85259, USA. ${ }^{3}$ The Biodesign Institute, Arizona State University, 1001 S. McAllister Ave, Tempe, AZ 85287, USA.

Received: 2 March 2013 Accepted: 17 January 2014

Published: 18 January 2014

\section{References}

1. Stupp R, Hegi ME, Mason WP, van den Bent MJ, Taphoorn MJ, Janzer RC, Ludwin SK, Allgeier A, Fisher B, Belanger K, et al: Effects of radiotherapy with concomitant and adjuvant temozolomide versus radiotherapy alone on survival in glioblastoma in a randomised phase III study: 5-year analysis of the EORTC-NCIC trial. Lancet Oncol 2009, 10(5):459-466.

2. Stupp R, Mason WP, van den Bent MJ, Weller M, Fisher B, Taphoorn MJ, Belanger K, Brandes AA, Marosi C, Bogdahn U, et al: Radiotherapy plus concomitant and adjuvant temozolomide for glioblastoma. N Engl J Med 2005, 352(10):987-996.

3. Hoelzinger DB, Mariani L, Weis J, Woyke T, Berens TJ, McDonough WS, Sloan A, Coons SW, Berens ME: Gene expression profile of glioblastoma multiforme invasive phenotype points to new therapeutic targets. Neoplasia 2005, 7(1):7-16.

4. Mariani L, Beaudry C, McDonough WS, Hoelzinger DB, Demuth T, Ross KR, Berens T, Coons SW, Watts G, Trent JM, et al: Glioma cell motility is associated with reduced transcription of proapoptotic and proliferation genes: a cDNA microarray analysis. J Neurooncol 2001, 53(2):161-176.

5. Demuth $T$, Rennert $J$, Hoelzinger DB, Reavie LB, Nakada M, Beaudry C, Nakada S, Anderson EM, Henrichs AN, McDonough WS, et al: Glioma cells on the run - the migratory transcriptome of 10 human glioma cell lines. BMC Genomics 2008, 9:54.

6. Joy AM, Beaudry CE, Tran NL, Ponce FA, Holz DR, Demuth T, Berens ME: Migrating glioma cells activate the PI3-K pathway and display decreased susceptibility to apoptosis. J Cell Sci 2003, 116(Pt 21):4409-4417.

7. Liu N, Okamura K, Tyler DM, Phillips MD, Chung WJ, Lai EC: The evolution and functional diversification of animal microRNA genes. Cell Res 2008, 18(10):985-996

8. Melo SA, Esteller M: Dysregulation of microRNAs in cancer: playing with fire. FEBS Lett 2011, 585(13):2087-2099.

9. Ciafre SA, Galardi S, Mangiola A, Ferracin M, Liu CG, Sabatino G, Negrini M, Maira G, Croce CM, Farace MG: Extensive modulation of a set of microRNAs in primary glioblastoma. Biochem Biophys Res Commun 2005, 334(4):1351-1358.

10. Lawler $\mathrm{S}$, Chiocca EA: Emerging functions of microRNAs in glioblastoma. J Neurooncol 2009, 92(3):297-306.

11. Novakova J, Slaby O, Vyzula R, Michalek J: MicroRNA involvement in glioblastoma pathogenesis. Biochem Biophys Res Commun 2009, 386(1):1-5.

12. Romero-Cordoba S, Rodriguez-Cuevas S, Rebollar-Vega R, Quintanar-Jurado V, Maffuz-Aziz A, Jimenez-Sanchez G, Bautista-Pina V, Arellano-Llamas R, Hidalgo-Miranda A: Identification and pathway analysis of microRNAs with no previous involvement in breast cancer. PloS One 2012, 7(3):e31904.

13. Papadopoulos GL, Alexiou P, Maragkakis M, Reczko M, Hatzigeorgiou AG: DIANA-mirPath: integrating human and mouse microRNAs in pathways. Bioinformatics 2009, 25(15):1991-1993. 
14. Reinhart BJ, Slack FJ, Basson M, Pasquinelli AE, Bettinger JC, Rougvie AE, Horvitz HR, Ruvkun G: The 21-nucleotide let-7 RNA regulates developmental timing in Caenorhabditis elegans. Nature 2000, 403(6772):901-906.

15. Pasquinelli AE, Reinhart BJ, Slack F, Martindale MQ, Kuroda MI, Maller B, Hayward DC, Ball EE, Degnan B, Muller P, et al: Conservation of the sequence and temporal expression of let-7 heterochronic regulatory RNA. Nature 2000, 408(6808):86-89.

16. Roush S, Slack FJ: The let-7 family of microRNAs. Trends Cell Biol 2008 18(10):505-516.

17. Wang X, Cao L, Wang Y, Wang X, Liu N, You Y: Regulation of let-7 and its target oncogenes (Review). Oncol Lett 2012, 3(5):955-960.

18. Lee ST, Chu K, Oh HJ, Im WS, Lim JY, Kim SK, Park CK, Jung KH, Lee SK, Kim $M$, et al: Let-7 microRNA inhibits the proliferation of human glioblastoma cells. J Neurooncol 2011, 102(1):19-24.

19. Johnson SM, Grosshans H, Shingara J, Byrom M, Jarvis R, Cheng A, Labourier $E$, Reinert KL, Brown D, Slack FJ: RAS is regulated by the let-7 microRNA family. Cell 2005, 120(5):635-647.

20. Shimizu S, Takehara T, Hikita H, Kodama T, Miyagi T, Hosui A, Tatsumi T, Ishida $H$, Noda T, Nagano $H$, et al: The let-7 family of microRNAs inhibits $\mathrm{BCl}-\mathrm{xL}$ expression and potentiates sorafenib-induced apoptosis in human hepatocellular carcinoma. J Hepatol 2010, 52(5):698-704.

21. Soni D, King JA, Kaye AH, Hovens CM: Genetics of glioblastoma multiforme: mitogenic signaling and cell cycle pathways converge. J Clin Neurosci 2005, 12(1):1-5.

22. Buechner J, Tomte E, Haug BH, Henriksen JR, Lokke C, Flaegstad T, Einvik C Tumour-suppressor microRNAs let-7 and mir-101 target the proto-oncogene MYCN and inhibit cell proliferation in MYCN-amplified neuroblastoma. Br J Cancer 2011, 105(2):296-303.

23. Shi L, Cheng Z, Zhang J, Li R, Zhao P, Fu Z, You Y: hsa-mir-181a and hsa-mir-181b function as tumor suppressors in human glioma cells. Brain Res 2008, 1236:185-193.

24. Zhu W, Shan X, Wang T, Shu Y, Liu P: miR-181b modulates multidrug resistance by targeting BCL2 in human cancer cell lines. Int J Cancer 2010, 127(11):2520-2529.

25. Xia L, Zhang D, Du R, Pan Y, Zhao L, Sun S, Hong L, Liu J, Fan D: miR-15b and miR-16 modulate multidrug resistance by targeting $B C L 2$ in human gastric cancer cells. Int J Cancer 2008, 123(2):372-379.

26. Fu M, Wang C, Li Z, Sakamaki T, Pestell RG: Minireview: cyclin D1: normal and abnormal functions. Endocrinology 2004, 145(12):5439-5447.

27. Wang J, Wang Q, Cui Y, Liu ZY, Zhao W, Wang CL, Dong Y, Hou L, Hu G, Luo $C$, et al: Knockdown of cyclin D1 inhibits proliferation, induces apoptosis, and attenuates the invasive capacity of human glioblastoma cells. J Neurooncol 2012, 106(3):473-484.

28. Shu J, Xia Z, Li L, Liang ET, Slipek N, Shen D, Foo J, Subramanian S, Steer CJ: Dose-dependent differential mRNA target selection and regulation by let-7a-7f and miR-17-92 cluster microRNAs. RNA Biol 2012, 9(10):1275-1287.

29. Bonci D, Coppola V, Musumeci M, Addario A, Giuffrida R, Memeo L, D'Urso L, Pagliuca A, Biffoni M, Labbaye C, et al: The miR-15a-miR-16-1 cluster controls prostate cancer by targeting multiple oncogenic activities. Nat Med 2008, 14(11):1271-1277.

30. Zhang H, Hao Y, Yang J, Zhou Y, Li J, Yin S, Sun C, Ma M, Huang Y, Xi JJ: Genome-wide functional screening of miR-23b as a pleiotropic modulator suppressing cancer metastasis. Nat Commun 2011, 2:554.

31. Pellegrino L, Stebbing J, Braga VM, Frampton AE, Jacob J, Buluwela L, Jiao $L R$, Periyasamy M, Madsen CD, Caley MP, et al: miR-23b regulates cytoskeletal remodeling, motility and metastasis by directly targeting multiple transcripts. Nucleic Acids Res 2013, 41(10):5400-5412

32. Loftus JC, Ross JT, Paquette KM, Paulino VM, Nasser S, Yang Z, Kloss J, Kim S, Berens ME, Tran NL: miRNA expression profiling in migrating glioblastoma cells: regulation of cell migration and invasion by miR-23b via targeting of Pyk2. PloS One 2012, 7(6):e39818.

doi:10.1186/1471-2105-15-21

Cite this article as: Bradley et al:: Differential expression of microRNAs as predictors of glioblastoma phenotypes. BMC Bioinformatics 2014 15:21.

\section{Submit your next manuscript to BioMed Central and take full advantage of:}

- Convenient online submission

- Thorough peer review

- No space constraints or color figure charges

- Immediate publication on acceptance

- Inclusion in PubMed, CAS, Scopus and Google Scholar

- Research which is freely available for redistribution 